\title{
Editorial
}

John M. Murkin MD FRCPC

\section{Monitoring cerebral oxygenation}

As exemplified by the clinical admonition beloved of many internists, "avoid hypoxia and hypotension," the brain is highly vulnerable to hypoxic or ischaemic insult. Even brief episodes of cerebral ischaemia e.g., ventricular tachycardia or fibrillation of $30 \mathrm{sec}$ duration, are sufficient to disrupt cerebral autoregulatory processes temporarily. ${ }^{1}$ Early studies in volunteers demonstrated loss of consciousness and simultaneous electroencephalographic (EEG) slowing within six to seven seconds of complete global ischaemia, followed by isoelectric EEG after 20-40 sec. ${ }^{2}$ Tonic clonic seizures and periods of transient postischaemic confusion follow ischaemic periods sustained for up to $100 \mathrm{sec}$. Concomitantly, cerebral oxygen levels are depleted within a very few seconds of the onset of ischaemia. Biochemical changes, including a $50 \%$ reduction in adenosine triphosphate (ATP) and an $85 \%$ decrease in phospho-creatinine (PCr), occur after $30 \mathrm{sec}$ global ischaemia in mice, ${ }^{3}$ with similar changes observed in human cerebral cortex. ${ }^{4}$ In mouse brain $60 \mathrm{sec}$ following decapitation, $\mathrm{PCr}$, glucose, and glycogen stores are less than $10 \%$, ATP is less than $25 \%$ of control, and lactate increases to $75 \%$ of its maximal value. ${ }^{5}$ In rats measurable decreases in $\mathrm{PCr}$ are seen after the first five seconds of ischaemia. ${ }^{6}$ Even brief periods of cerebral ischaemia can thus be seen to induce profound functional and metabolic changes in animals as well as in man.

With rare exception, patients undergoing anaesthesia and surgery are well served by general clinical measures designed to limit the occurrence of cerebral hypoxia/ ischaemia. Nonetheless, because of the exquisite sensitivity of the brain to anoxia, certain clinical situations may predispose towards, or even potentially exacerbate, brain injury. For example, the management of patients with increased intracranial pressure (ICP), those undergoing certain neurosurgical or cardiac surgical procedures, or patients with head injury, often involves techniques that can profoundly influence both cerebral blood flow (CBF) and cerebral metabolic rate (CMR), altering the cerebral milieu in a potentially unpredictable manner. The introduction of various newer monitoring modalities

From the University Hospital, University of Western Ontario, London, Ontario. and techniques into the clinical armamentarium is now enabling a closer assessment of cerebral oxygen balance to be made. The metabolic consequences for the brain of diverse clinical techniques including hyperventilation, pharmacological suppression of EEG activity, hypothermia/rewarming, retrograde cerebral perfusion, etc., are now able to be assessed and monitored clinically, outside the laboratory setting.

In this issue of the Journal, Matta et al. report on the use of transcranial Doppler (TCD) and intermittent sampling of cerebral venous blood, to assess cerebral oxygenation during hyperventilation and graded hyperoxia. ${ }^{7}$ They investigated neurosurgical patients undergoing craniotomy and, while their study may be subject to criticism regarding methodology and technique, (e.g., measuring flow velocity rather than flow, apparent use of calculated rather than measured blood oxygen saturation, intermittent rather than continuous measures of saturation), it nevertheless provides valuable clinical insights. At specific issue was whether the increase in blood oxygen content achieved by hyperoxia would be offset or negated by an augmented cerebral vasoconstrictive response, whereby both hyperoxia and hypocapnia would act to decrease $\mathrm{CBF}$ resulting in no change, or even a net reduction, in cerebral oxygen delivery. By determining jugular venous oxygen saturation $\left(\mathrm{SjO}_{2}\right)$ and cerebral blood flow velocity, they were able to address this issue, demonstrating that an augmented cerebral vasoconstrictive response did not occur. Their results can be interpreted as reinforcing the considered clinical reaction to potential hypoxia, an increase in inspired oxygen content, as being sound even in the context of variable and potentially competing cerebrovascular responses. One implication of this study is that it provides further evidence of the increasing utilization of monitoring techniques that can help guide and refine clinical practice in those instances where cerebral well-being is at issue, analogous to the monitors now widely employed for assessing indices of myocardial function.

In addition to helping to guide therapy in neurosurgical patients, as reported in this issue, other clinical applications of jugular sinus cannulation have involved both adult and paediatric patients undergoing cardiac surgical 
procedures, as well as encephalopathic and head-injured patients treated in an intensive care setting. Jugular oximetry has been used to help to define the influence of $\mathrm{pH}$ management during hypothermic cardiopulmonary bypass (CPB), ${ }^{8}$ measure the cerebrovascular and metabolic responses to hypothermic circulatory arrest, ${ }^{9}$ assess cerebral oxygenation during hyperventilation in patients with increased ICP, ${ }^{10}$ determine the adequacy of cerebral perfusion pressure in head injury, ${ }^{11}$ among other uses. For these applications and others, monitoring and interpretation of variables derived from jugular bulb cannulation necessitates some understanding of cerebral anatomy and physiology.

After collecting in the sagittal and straight sinuses, cerebral venous blood drains into the jugular sinuses via the sigmoid sinuses and thence into the jugular bulb at the base of the skull. Based on dye-injection experiments, it is estimated that less than $3 \%$ of blood obtained from the superior bulb of the internal jugular vein drains extracerebral tissues, largely the result of communications from the small mastoid and parietal emissary veins, and the basilar and pterygoid plexuses. ${ }^{12}$ Due to mixing of hemispheric blood in the straight and superior sagittal sinuses, only about two-thirds of blood supplied to one hemisphere through an internal carotid artery is drained through the ipsilateral internal jugular vein. Ipsilateral jugular sinus blood is thus largely representative of bihemispheric mixed cerebral venous blood, rather than ipsilateral hemispheric flow. ${ }^{13}$ There is some evidence that blood within the right versus the left jugular bulb may not be equally representative of all brain regions, however. ${ }^{14}$ In the absence of intracranial pathology, blood from the straight sinus drains preferentially into the left jugular bulb, which is thus potentially weighted more towards subcortical structures, whereas blood from the sagittal sinus drains more selectively into the right jugular bulb and is thus correspondingly more representative of venous blood from cortical areas. Which side to cannulate and monitor is thus controversial. In the presence of head injury or increased ICP, jugular cannulation ipsilateral to the lesion is practiced by some. ${ }^{15}$ Others recommend observing the effect on ICP of unilateral compression of each jugular vein, and cannulation of the side producing the largest rise in ICP. "

Determining appropriate catheter location not only involves selecting which side should be cannulated, but also ensuring correct placement of the catheter tip within the bulb of the jugular sinus. In the original, classic studies of Kety and Schmidt, CBF was measured using direct needle cannulation of the jugular bulb immediately below the mastoid process. ${ }^{16}$ Subsequently, this technique has been modified to allow retrograde cannulation of the jugular bulb from a site lower in the neck, generally near the level of the cricoid cartilage. ${ }^{17,18}$ This necessitates radiographic confirmation of correct location of the tip of the catheter within the jugular bulb, i.e., above the level of the second cervical vertebra on lateral cervical $x$-ray. Additionally, the rate of withdrawal for blood sampling must be sufficiently gradual to minimize extracerebral contamination from retrograde flow via the facial vein which drains into the jugular vein within 2 to $5 \mathrm{~cm}$ of its exit from the skull. ${ }^{12}$ With these provisos, it can be seen that analysis of jugular sinus blood can provide valuable information regarding status of global cerebral oxygenation in a variety of clinical situations.

In addition to intermittent blood sampling, developments in intravascular fibreoptic technology employing near infrared spectrophotometry (NIRS) techniques enable blood oxygen saturation to be monitored continuously. Light transmission through tissue depends upon a combination of reflectance, scattering and absorption effects, favouring transmission of near infrared (650-1100 $\mathrm{nm}$ ) light. Absorption occurs at specific wavelengths, determined by the molecular properties of the materials in the light path. Organometallic molecules such as haemoglobin ( $\mathrm{Hb}$ ) and cytochrome aa3 (Caa3), are lightabsorbing molecules (chromophores) that have wellcharacterized infrared absorption spectra which shift with oxygenation, allowing spectrophotometric analysis. ${ }^{19} \mathrm{Jug}$ ular cannulation with a fibreoptic NIRS catheter has been used for continuous monitoring of $\mathrm{SjO}_{2}$ in various clinical situations. While originally employed for monitoring paediatric umbilical artery oxygen saturations, subsequent studies have demonstrated good correlation over a range of venous oxygen saturations from $89.6 \%$ to as low as 9.4\%. ${ }^{20}$ During CPB, good correlation was also demonstrated with in vitro co-oximetry measurements during haemodilution, normothermia and hypothermia. ${ }^{21}$ These studies demonstrate excellent correlation between in vivo and in vitro measurements of blood oxygen saturation over the extremes of physiological variability, and reinforces the potential utility of jugular co-oximetry as a clinical monitor. Again, verification of appropriate positioning of the catheter tip within the jugular bulb using radiography, ultrasonography, or other techniques, is essential. In addition, in using continuous intravascular NIRS, impingement of catheter tip on vessel wall may cause inappropriate light reflection (wall artifact) due to backscatter from the wall rather than from haemoglobin, manifesting as abnormal light intensity, and requiring repositioning of the catheter.

While a potential limitation of $\mathrm{SjO}_{2}$ is the relative insensitivity to regional desaturation as a consequence of the global nature of mixed cerebral venous blood, conversely, newer noninvasive oximetry devices may be limited by their highly selective regional nature. Noninvasive 
devices employing reflectance NIRS for continuous monitoring of cerebral oxygenation have recently been undergoing clinical assessment. These employ principles of optical spectrophotometry exploiting the fact that biological material is relatively transparent in the near infrared range. Near infrared light penetrates tissue several centimeters. ${ }^{19}$ Percutaneous reflectance NIRS utilizes the reflected characteristic of incident light by employing ipsilateral placement of both light source and sensor. Additionally, by using both a "shallow" and a "deep" sensor configuration, the effect of extracranial tissue can be effectively neutralized by subtraction techniques. ${ }^{22}$ In this application, because of difficulties in quantitating absorption of individual spectra, the ratio of haemoglobin to oxyhaemoglobin, calculated as cerebrovascular oxygen saturation, is determined. The accuracy of this estimate is influenced by the partitioning of cerebral blood volume into arterial, venous and capillary compartments. Weighted $70-80 \%$ towards venous blood, the analysis algorithm employed derives a value that represents regional. cerebral tissue oxygen saturation $\left(\mathrm{SrO}_{2}\right) \cdot{ }^{23}$ Changes in intracranial blood distribution, e.g., subdural haematoma, may thus decrease the accuracy of $\mathrm{SrO}_{2}$ as a result of changes in the relative size of each vascular compartment. In addition, with the usual percutaneous sensor placement over the region of the frontal lobe, the volume of brain tissue sampled is approximately $1.0-1.5 \mathrm{ml}$ at a depth of 8 to $12 \mathrm{~mm}$, thus the resultant $\mathrm{SrO}_{2}$ is highly localized. ${ }^{22}$ This technique has been assessed both by using hypoxic challenge in volunteers, wherein $\mathrm{SrO}_{2}$ demonstrated earlier detection of hypoxia than did EEG, and by demonstrating good correlation of $\mathrm{SrO}_{2}$ with jugular bulb saturation in head-injured patients. ${ }^{22}$ Although $\mathrm{SrO}_{2}$ was shown to be highly sensitive in the presence of global hypoxia or ischaemia, the occurrence of localized cerebral ischaemia outside the small area of brain being monitored may still go undetected, though for a fundamentally different reason than that which causes $\mathrm{SjO}_{2}$ to be insensitive. Further clinical studies to define the role of $\mathrm{SrO}_{2}$ monitoring are awaited.

The ability to monitor cerebral oxygenation readily on either an intermittent or a continuous basis is now at hand. Whether these techniques will reinforce established clinical practice, or lead to the development of novel cerebroprotective strategies, remains to be seen. Regardless of these larger issues, however, closer monitoring and titration of brain oxygenation in individual patients will allow further optimization of care for the brain at risk.

\section{Le monitorage de l'oxygénation cérébrale}

Conformément au dicton qui énonce qu'il faut " éviter l'hypoxie et l'hypotension " que nous servent si souvent les internistes, le cerveau est très vulnérable à l'outrage de l'hypoxie et de l'ischémie. Même les plus brefs épisodes d'schémie cérébrale comme la tachycardie et la fibrillation ventriculaires réussissent à interrompre le mécanisme de l'autorégulation cérébrale.' Des études réglées menées chez des volontaires ont montré que la perte de conscience et la ralentissement électroencéphalographique (EEG) simultané survenant en deça de six à sept sec de l'ischémie complète étaient suivi d'un EEG isoélectrique après 20 à $40 \mathrm{sec}^{2}$ Des convulsions toniques et des périodes de confusion ischémiques transitoires suivaient des épisodes d'ischémie maintenue jusqu'à $100 \mathrm{sec}$. Simultanément, la réserve cérébrale d'oxygène s'épuise après quelques secondes d'ischémie. Chez la souris, après $30 \mathrm{sec}$ d'ischémie globale, des changements biochimiques surviennent dont une déplétion de $50 \%$ de l'adénosine triphosphate (ATP) et une chute de $85 \%$ de la phosphocréatinine $(\mathrm{PCr}),{ }^{3}$ ce qui comparable à ce qui survient au cortex cérébral humain. ${ }^{4}$ Au niveau du cerveau de la souris, $60 \mathrm{sec}$ après décapitation, la $\mathrm{PCr}$, le glucose, et les réserves de glycogène baissent à moins de $10 \%$, l'ATP diminue sous $25 \%$ du contrôle et le lactate augmente à $75 \%$ de sa valeur maximale. ${ }^{5}$ Chez le rat, des baisses tangibles de $\mathrm{PCr}$ sont notées après cinq secondes d'ischémie. ${ }^{6}$ Les plus courtes périodes d'ischémie provoquent donc des changements métaboliques et fonctionnels graves tant chez l'animal que chez l'homme.

En général, grâce aux mesures orientées vers la prévention de l'hypoxie et de l'ischémie cérébrales, on peut affirmer que les patients sous anesthésie sont protégés. Cependant, à cause de la sensibilité extrême du cerveau à l'anoxie, certaines situations peuvent provoquer, ou même favoriser les dommages cérébraux. Par exemple, les patients souffrant d'hypertension intracrânienne, certains patients qui subissent des interventions neurochirurgicales ou cardiaques et les traumatisés du crâne sont soumis à des techniques qui peuvent influer autant sur le débit sanguin cérébral (DSC) que sur son métabolisme (MC) et altérer le milieu cérébral de façon imprévisible. Lintroduction de nouvelles méthodes et techniques de surveillance nous permet une meilleure évaluation de l'équilibre en oxygène. Les conséquences cérébrales métaboliques de quelques techniques dont l'hyperventilation, l'inhibition métabolique de l'activité EEG, l'hypothermie 
et le réchauffement, la perfusion cérébrale rétrograde etc., peuvent maintenant être évaluées et monitorées en dehors du laboratoire.

Dans ce numéro du Journal, Matta et al. utilisent le Döppler transcrânien (DTC) et l'échantillonnage itératif du sang veineux cérébral pour évaluer l'oxygénation du cerveau pendant l'hyperventilation et l'hyperoxie progressive. ${ }^{7}$ Les auteurs évaluent des patients soumis à la une craniotome, et, bien que leur étude soit contestable autant sous l'aspect méthodologique que technique (par ex., par la mesure de la vélocité du flux à la place de la mesure du débit, par l'utilisation de valeurs de saturation calculées plutôt que mesurées), elle présente sur le plan clinique une importance significative. Spécifiquement, on se demandait si l'augmentation du contenu en oxygène du sang obtenu pendant l'hyperoxie serait nulle ou même dommageable par l'augmentation réflexe de la vasoconstriction, alors que l'hyperoxémie associée à l'hypocapnie provoquerait la chute du DSC, avec comme conséquence l'absence de changement ou même une nette réduction de l'apport d'oxygène au cerveau. En déterminant la saturation veineuse au bulbe jugulaire $\left(\mathrm{SjO}_{2}\right)$, ils ont réussi à aborder ce problème et montrer que l'exagération de la réponse vasoconstrictrice ne survenait pas. Leurs résultats peuvent être interprétés comme un argument en faveur de l'impression selon laquelle une augmentation du contenu oxygéné est bénéfique même dans un contexte de réponses cérébrovasculaires inconstantes et possiblement opposées. Un corollaire de cette étude est qu'il est prouvé une fois de plus qu'il faut faire appel à des techniques de monitorage là où elles peuvent diriger et raffiner la gestion des situations où la survie cérébrale est en jeu, de la même façon qu'on les utilisent pour évaluer les indices de performance myocardique.

En plus de favoriser le traitement neurochirurgical, on peut appliquer la technique de la canulation du sinus jugulaire aussi bien aux adultes et aux enfants soumis à la chirurgie cardiaque qu'aux patients de l'unité des soins intensifs souffrant d'encéphalopathie et de traumatisme crânien. L'oxymétrie jugulaire a été utilisée, entre autres, pour préciser l'influence de la gestion du $\mathrm{pH}$ pendant la $\mathrm{CEC},{ }^{8}$ pour mesurer les réponses cérébrovasculaires et métaboliques à l'arrêt circulatoire hypothermique, ${ }^{9}$ pour évaluer l'oxygénation cérébrale pendant l'hyperventilation pour hypertension intracrânienne, ${ }^{10} \mathrm{et}$ pour évaluer l'efficacité de la pression de perfusion cérébrale chez les traumatisés du crâne. " Le monitorage et l'interprétation des variables obtenues par la canulation du bulbe jugulaire exige toutefois une connaissance de l'anatomie et de la physiologie cérébrales.

Après sa collection dans les sinus sagittaux et rectilignes, le sang veineux cérébral draine dans les sinus jugulaires via les sinus sigmoïdes et de là parvient au bulbe jugulaire à la base du crâne. Grâce à des expériences avec des colorants, on estime que moins de $3 \%$ du sang recueilli au bulbe supérieur de la jugulaire interne draine le tissu extracérébral, en grande partie à cause de communications avec les petites veines mastoïdiennes et pariétales émissaires et avec les plexus basilaires et ptérygoïdes. ${ }^{12}$ Par mixage du sang hémisphérique dans les sinus rectilignes et sagittaux supérieurs, seulement les deux tiers du sang acheminé à un hémisphère par la carotide interne draine par la veine jugulaire interne homolatérale. Le sang veineux jugulaire représente donc avec assez de précision la composition du sang veineux mêlé cérébral bi-hémisphérique, plutôt que le contenu homolatéral du sang hémisphérique. ${ }^{13} \mathrm{Il}$ semble toutefois que le sang drainé du côté droit comparativement à celui qui draine au bulbe jugulaire gauche ne soit pas typique de toutes les régions du cerveau. ${ }^{14}$ En absence de lésion intracrânienne, le sang des sinus rectilignes draine de préférence dans le bulbe jugulaire gauche, donc provient surtout des structures sous-corticales alors que le sang des sinus sagittaux draine plus sélectivement dans le bulbe jugulaire droit et correspond surtout au sang des zones corticales. En présence d'un traumatisme cérébral ou d'une augmentation de la pression intracrânienne (PIC), certains pratiquent la canulation jugulaire du même côté que la lésion. ${ }^{15}$ D'autres recommandent d'observer le résultat de la compression uniliatérale de chacune des jugulaires sur la PIC, et de canuler le côté qui produit la plus grande élévation de la PIC."

Bien positionner le cathéter ne signifie pas seulement choisir le bon côté, mais aussi confirmer que l'extrémité du cathéter est situé dans le bulbe carotidien. Dans les études classiques de Kety et Schmidt, le DSC était mesuré par la canulation directe à l'aiguille du bulbe jugulaire immédiatement sous la mastoïde. ${ }^{16} \mathrm{Par}$ la suite, cette technique a été modifiée pour la canulation rétrograde du bulbe jugulaire plus bas dans le cou, ordinairement au niveau du cartilage cricoïde. ${ }^{17,18}$ Cette manoeuvre nécessite une confirmation radiographique, c'est-à-dire que l'extrémité du cathéter doit se retrouver au niveau supérieur de la deuxième vertèbre cervicale sur une radiographie latérale du cou. De plus, le prélèvement doit être graduel pour minimiser la contamination extracérébrale du flux rétrograde provenant de la veine faciale à 2 à $5 \mathrm{~cm}$ de sa sortie de la boite crânienne. ${ }^{12}$ Avec ces précautions, on peut présumer que l'analyse du sang jugulaire sinusal procurera des renseignements valides sur l'état de l'oxygénations globale cérébrale dans une grande variété de situations.

En plus de l'étalonnage sanguin itératif; on peut maintenant, grâce à la technologie fibroptique intravasculaire avec spectophotométrie de réflexion qui utilise des longueurs d'ondes voisines de l'infrarouge (SPR) monitorer 
en continu la saturation en oxygène. La transmission lumineuse transtissulaire dépend d'une combinaison des effets de la réflectance, de la dispersion et de l'absorption dans une bande voisine de la lumière infrarouge $(650-1100 \mathrm{~nm})$. L'absorption se produit à des longueurs d'ondes spécifiques déterminées par les propriété moléculaires de la substance que la lumière traverse. Les molécules organo-métaliques comme l'hémoglobine $(\mathrm{Hb})$ et le cytochrome aa3 (Caa3) sont des molécules qui absorbent la lumière (chromophores); elles possèdent des spectres précis d'absorption qui dévient avec l'oxygénation et ainsi se prêtent bien à l'analyse par SPR. ${ }^{19}$ On a utilisé la canulation jugulaire pour le monitorage en continu de la $\mathrm{SjO}_{2}$ dans plusieurs situations. Originalement utilisée pour le monitorage de la saturation artérielle ombilicale en néonatalogie, des études subséquentes ont montré une bonne corrélation sur une fourchette étendue de valeurs allant de $89,6 \%$ jusquà $9,4 \% .{ }^{20}$ Pendant la CEC, la corrélation est bonne avec des mesures de cooxymétrie in vitro pendant l'hémodilution, la normothermie et l'hypothermie. ${ }^{21}$ Ces études ont démontré l'excellente corrélation des mesures in vivo et in vitro de la saturation dans les extrêmes de la variabilité physiologique, permettant ainsi l'utilisation éventuelle du cooxymètre jugulaire comme moniteur. Encore là, la vérification de la position de l'extrémité du cathéter au bulbe jugulaire par radiographie, ultrasonographie ou d'autres techniques est essentielle. De plus pendant l'utilisation de la SPR, l'empiétement du bout du cathéter sur la paroi du vaisseau peut causer une réflexion inappropriée de la lumière (artéfact de paroi) due à la dispersion sur la paroi arrière, plutôt que sur l'hémoglobine, se manifestant comme une intensité lumineuse anormale, et nécessitant une repositionnement du cathéter.

Alors que la limitation de la $\mathrm{SjO}_{2}$ vient de sa relative insensibilité à la désaturation régionale à cause de la nature globale du sang veineux cérébral mêlé, au contraire, les nouveaux appareils non effractifs oxymétriques peuvent être limités par leur particularités de sélectivité régionale. Des appareils non effractifs utilisant la SPR pour le monitorage continu de l'oxygénation cérébrale ont été récemment testés. Ils utilisent les principes de la spectrophotométrie optique suivant lesquels la matière biologique est relativement transparente dans un éventail d'ondes voisines des infrarouges. La lumière infrarouge pénètre plusieurs centimètres dans les tissus. ${ }^{19}$ La SPR par réflectance percutanée utilise les caractéristiques de réflexion de la lumière incidente par positionnement homolatéral de la source lumineuse et du senseur. De plus, en utilisant les configurations d'un senseur superficiel et d'un senseur profond, on peut par soustraction éliminer l'effet du tissus extracrânien. ${ }^{22}$ Dans ce montage, à cause des problèmes de quantification de l'absorption des spectres individuels, le rapport entre l'hémoglobine et l'oxyhémoglobine, calculé comme la saturation cérébrale en oxygène, est déterminé. La précision de cette évaluation est influencée par la répartition du volume sanguin cérébral en compartiments artériel, veineux et capillaire. Présumant un pourcentage de $70-80 \%$ en sang veineux, l'organigramme analytique utilisé déduit une valeur qui représente la saturation cérébrale régionale $\left(\mathrm{SrO}_{2}\right) \cdot{ }^{23}$ Les changements intracrâniens de distribution du sang causés, par exemple, par un hématome sous-dural, peuvent ainsi diminuer la précision de la $\mathrm{SrO}_{2}$ en changeant la dimension relative de chacun des compartiments vasculaires. De plus avec le senseur percutané usuel positionné sur la région du lobe frontal, le volume de tissus analysé est d'environ 1,0-1,5 ml à la profondeur de 8 à $12 \mathrm{~mm}$, donc une analyse très localisée. ${ }^{22}$ Cette technique a été évaluée par provocation hypoxique chez des volontaires, alors que la $\mathrm{SrO}_{2}$ détecte l'hypoxie plus précocement que l'EEG, et par la démonstration de la corrélation entre la $\mathrm{SrO}_{2}$ et la saturation du bulbe jugulaire chez des traumatisés crâniens. ${ }^{22}$ Bien qu'on ait réussi à démontrer la sensibilité élevée de la $\mathrm{SrO}_{2}$ en présence d'hypoxie et d'ischémie globales, l'apparition d'une ischémie cérébrale localisée survenant en dehors de la petite zone monitorée peut échapper à la détection, bien que ce soit pour une raison fondamentalement différente que celle qui cause l'insensibilité de la $\mathrm{SjO}_{2}$. Des études ultérieures seront requises pour définer le rôle précis du monitorage de la $\mathrm{SrO}_{2}$.

La capacité de monitorer l'oxygénation immédiate du cerveau, que ce soit de façon intermittente ou continue, est maintenant à notre portée. On ne peut affirmer maintenant que ces techniques vont améliorer la pratique clinique ou amener le développement de nouvelles stratégies de protection cérébrale. Cependant à défaut de réalisations dont la portée pourrait être plus vaste, le monitorage et le contrôle plus étroits de l'oxygénation cérébrale conduira à l'amélioration des soins lorsque l'intégrité du cerveau est menacée.

\section{References}

1 Murkin JM. Cerebrovascular reactivity after transient circulatory arrest. Stroke 1992; 23 (Suppl 1): 3.

2 Rossen $R$, Kabat $H$, Anderson JP. Acute arrest of cerebral circulation in man. Arch Neurol Psychiat 1943; 50: 510-28.

3 Goldberg ND, Passonneau JV, Lowry OH. Effects of changes in brain metabolism on the levels of citric acid cycle intermediates. J Biol Chem 1966; 241: 3997-4003.

4 Kirsch WM, Leitner JW. Glycolytic metabolites and cofactors in human cerebral cortex and white matter during complete ischemia. Brain Res 1967; 4: 358-68. 
5 Lowry OH, Passonneau JV. The relationship between substrates and enzymes of glycolysis in brain. J Biol Chem 1964; 239: 31-42.

6 Winn HR, Rubio R, Berne RM. Brain adenosine production in the rat during 60 seconds of ischemia. Circ Res 1979; 45: 486-92.

7 Matta BF, Lam AM, Mayberg TS. The influence of arterial oxygenation on cerebral venous oxygen saturation during hyperventilation. Can J Anaesth 1994; 41: 1041-6.

8 Murkin JM, Farrar JK, Tweed WA, McKenzie FN, Guiraudon $G$. Cerebral autoregulation and flow/metabolism coupling during cardiopulmonary bypass: the influence of $\mathrm{PaCO}_{2}$. Anesth Analg 1987; 66: 825-32.

9 Greeley WJ, Kern FH, Ungerleider RM, et al. The effect of hypothermic cardiopulmonary bypass and total circulatory arrest on cerebral metabolism in neonates, infants, and children. J Thorac Cardiovasc Surg 1991; 101: 783-94.

10 Obrist WD, Langfitt TW, Jaggi $J L$, Cruz J, Gennarelli TA. Cerebral blood flow and metabolism in comatose patients with acute head injury. Relationship to intracranial hypertension. J Neurosurg 1984; 61: 241-53.

11 Dearden $N M . \mathrm{SjO}_{2}$ and critical cerebral perfusion pressure after severe brain injury. British Journal of Intensive Care 1992; Suppl 1: 7-11.

12 Shenkin HA, Harmel MH, Kety SS. Dynamic anatomy of the cerebral circulation. AMA Archives of Neurology and Psychiatry 1948; 60: 240-52.

13 Gibbs $E L$, Lennox $W G$, Gibbs $F A$. Bilateral internal jugular blood. Comparison of A-V differences, oxygen-dextrose ratios and respiratory quotients. Am J Psychiatry 1945; 102: 184-9.

14 Dearden NM. Jugular bulb venous blood oxygen saturation in the management of severe head injury. Current Opinions in Anaesthesiology 1991; 4: 279-86.

15 Robertson CS, Narayan RK, Gokaslan $Z L$, et al. Cerebral arteriovenous oxygen difference as an estimate of cerebral blood flow in comatose patients. J Neurosurg 1989; 70: 222-30.

16 Kety SS, Schmidt CF. The determination of cerebral blood flow in man by the use of nitrous oxide in low concentrations. Am J Physiol 1945; 143: 53-66.

17 Maekawa T, Sakabe T. An easy technique for catheterization of the internal jugular bulb (Letter). Stroke 1984; 15: 179-80.

18 Gayle MO, Frewen TC, Armstrong RF, et al. Jugular venous bulb catheterization in infants and children. Crit Care Med 1989; 17: 385-8.

19 Gettinger A, DeTraglia MC, Glass DD. Accuracy of two mixed-venous saturation catheters. Anesthesiology 1986; 56: Al45.

20 Nakajima $T$, Ohsumi H, Kuro $M$. Accuracy of continuous jugular bulb venous oximetry during cardiopulmonary bypass. Anesth Analg 1993; 77: 1111-5.
21 Jobsis FF. Noninvasive, infrared monitoring of cerebral and myocardial oxygen sufficiency and circulatory parameters. Science 1977; 198: 1264-7.

22 McCormick PW, Stewart M, Goetting MG, Balakrishnan $G$. Regional cerebrovascular oxygen saturation measured by optical spectroscopy in humans. Stroke 1991; 22: 596-602.

23 McCormick $P W$, Stewart $M$, Lewis $G$. Noninvasive measurement of regional cerebrovascular oxygen saturation in humans using optical spectroscopy. International Society for Optical Engineering Proceedings; 1991. 